\title{
$\angle S$ Research Square \\ Establishing and Assessing a Nomogram Based on the Clinical Features of Mucosal Melanoma of the Head and Neck
}

Qing-Qing Xu

Sun Yat-sen University Cancer Center

Qing-Jie Li

Sun Yat-sen University Cancer Center

Liu Chen

Sun Yat-sen University Cancer Center

Xin-Yi Su

Sun Yat-sen University Cancer Center

Jing-Xia Song

Sun Yat-sen University Cancer Center

Juan Du

Hubei Cancer Hospital

Lei Chen

Sun Yat-sen University Cancer Center

Li-Xia Lu ( $\nabla$ lulx@sysucc.org.cn )

Sun Yat-sen University Cancer Center https://orcid.org/0000-0002-0549-3786

\section{Primary research}

Keywords: mucosal melanoma of the head and neck, clinical outcomes, prognostic factors, nomogram

Posted Date: February 23rd, 2021

DOI: https://doi.org/10.21203/rs.3.rs-230826/v1

License: (1) (1) This work is licensed under a Creative Commons Attribution 4.0 International License. Read Full License

Version of Record: A version of this preprint was published at Cancer Cell International on April 17th, 2021. See the published version at https://doi.org/10.1186/s12935-021-01927-7. 


\section{Abstract}

Objectives: The rarity of mucosal melanoma of the head and neck (MMHN) and the lack of prospective clinical trials has resulted in the limited knowledge of its clinical features and prognosis. We aimed to understand the clinical characteristics and developed a nomogram to better predict the prognosis of patients with MMHN.

Methods: Based on a total of 300 patients with nonmetastatic MMHN, multivariable Cox regression was performed to analyze independent prognostic factors. The overall survival (OS), disease-free survival (DFS), distant metastasis-free survival (DMFS), and locoregional relapse-free survival (LRRFS) of MMHN patients could be monitored using the nomogram. To facilitate clinical application, an online dynamic nomogram was established.

Results: Multivariate analysis identified primary tumor site, $\mathrm{T}$ stage and $\mathrm{N}$ stage as independent risk factors for survival. This factor-based nomogram had prognostic value for OS, DFS, DMFS, and LRRFS. Receiver operating characteristic analysis indicated the high diagnostic accuracy of the nomogram (AUC $>0.7)$. Kaplan-Meier survival curves indicated that the risk score of the nomogram effectively stratified MMHN patients with poor survival into a high-risk group (all $\mathrm{P}<0.001$ ).

Conclusions: The nomogram is conducive to stratifying MMHN patients into clinically meaningful taxonomies and subsequently providing individualized treatment.

\section{Introduction}

Mucosal melanoma of the head and neck (MMHN) is a highly malignant tumor accounting for more than $50 \%$ of all mucosal melanomas ${ }^{1}$ and is mainly found in the nasal cavity, paranasal sinuses and oral cavity ${ }^{2}$. Compared with cutaneous and acral melanomas, mucosal melanoma has aggressive clinical characteristics and a worse prognosis ${ }^{3-5}$. Despite the application of various clinical treatments, including surgery, radiotherapy, chemotherapy and immunotherapy, the local control and long-term prognosis of MMHN remain quite dismal and seem to show no improvement trends in recent years ${ }^{6,7}$. Due to its rarity, there is no effective prognostic tool available for MMHN. Therefore, more accurate prognostic tools are needed in addition to improving the treatment.

Considering the rarity of MMHN and the paucity of optimal treatment modalities following diagnostic tools, in this study, we included the largest single-center sample size, aiming to further explore MMHN by analyzing the clinical features and treatment outcomes. A nomogram serves as a new reliable tool for predicting the prognosis of cancers ${ }^{8-10}$. Therefore, we combined the TNM staging system and clinical features to develop a nomogram to accurately predict the survival outcomes of MMHN patients to facilitate clinical decision making.

\section{Materials And Methods}




\section{Patients}

From March 1986 to November 2019, three hundred patients diagnosed with MMHN in our hospital were included in the primary cohort. MMHN patients from March 1986 to December 2014 were chosen as the validation cohort. The inclusion criteria for all patients were previously untreated, nonmetastatic, and newly histologically confirmed stage III-IVB MMHN. All patients were restaged according to the 8th edition American Joint Committee on Cancer (AJCC) staging system for MMHN ${ }^{11}$. The TNM classification was based on surgical documents, pathological features and imaging findings. The exclusion criteria were as follows: (1) distant metastases before the treatment, secondary malignancy, or both; (2) pregnancy or lactation; and (3) incomplete previous medical records, auxiliary examinations, and follow-up information. The Ethics Committee at Sun Yat-sen University Cancer Center in China approved our study protocol.

\section{Follow-up and Endpoints}

Our main endpoint was overall survival (OS), and the secondary endpoints were disease-free survival (DFS), distant metastasis-free survival (DMFS), and locoregional relapse-free survival (LRRFS). OS was defined as the time from the diagnosis of melanoma to the date of death or the last known follow-up, whichever occurred first; DMFS was defined as the time to distant metastasis, death, or patient censoring, whichever occurred first; DFS was defined as the time to failure, death from any cause, or patient censoring, whichever occurred first; and LRRFS was defined as the time to local/regional relapse, death, or patient censoring, whichever occurred first. The median follow-up time was 32 months (range: 1-262 months). After treatment, the patients were evaluated once every 3 months during the first 3 years and every 6 months thereafter.

\section{Statistical Analysis}

The patients were classified into 2 groups based on age ( $<60$ years vs. $\geq 60$ years). Variables satisfying $P<0.1$ in univariate Cox regression analysis were included in the multivariable analysis. $P<0.05$ in multivariable Cox regression analysis was used to select independent prognostic variables of survival. All independent prognostic factors were used to create a predictive nomogram (by the rms package in $\mathrm{R}$ ). The concordance index (c-index) values with 95\% confidence intervals (Cls) were evaluated to assess the accuracy of the nomogram in the primary and validation cohorts. Calibration plots for OS, DMFS, and DFS at three and five years were generated by comparing the predicted OS, DMFS, and DFS with the actual OS, DMFS, and DFS. Moreover, the predictive precision and discrimination of the nomogram were further analyzed by the Akaike information criterion (AIC), c-index, and area under the curve (AUC) of the receiver operating characteristic $(\mathrm{ROC})$ curve. Survival curves were generated using the Kaplan-Meier method. Differences in survival between risk groups were analyzed by the log-rank test. The statistical analysis was performed with $\mathrm{R}$ software (R version 3.6.1) (http://www.r-project.org) and IBM SPSS software version 25.0 .

\section{Results}




\section{Patient Characteristics and Follow-up}

From March 1986 to November 2019, 300 patients (primary cohort) and 182 patients (validation cohort) with MMHN were enrolled in this study. The median age was 57 (range, 19-87) years with a male-tofemale ratio of 1.54:1 for the primary cohort. The median age was 58 (range, 25-80) years with a maleto-female ratio of $1.54: 1$ for the validation cohort. The baseline patient characteristics of the primary cohort and validation cohort are listed in Table 1. 
Table 1

Comparison of the different characteristics between patients in the primary and validation cohorts.

Characteristic
Number of patients (\%)

\section{Primary patients}

$(n=300)$

$P$-value

\section{Validation cohort}

$(n=182)$

\section{Gender}

Male

Female

Age (years old)

$<60$

$\geq 60$

Smoking

No

Yes

Primary site

Others

Nasal cavity

Paranasal sinus

Oral cavity

8th T stage

T3

$\mathrm{T} 4 \mathrm{a}$

$\mathrm{T} 4 \mathrm{~b}$

8th $\mathrm{N}$ stage

NO

N1

Surgery

182 (60.7)

118 (39.3)

174 (58.0)

126 (42.0)

220 (73.3)

$80(26.7)$
23 (12.6)

$100(54.9)$

$11(6.0)$

48 (26.4)
73 (40.1)

77 (42.3)

32 (17.5)

50 (16.7)

0.604

132 (72.5)

$50(27.5)$
0.944

0.373

0.064

0.753

$$
0.966
$$

121 (40.3)

224 (74.7)

$76(25.3)$

0.933

Abbreviations:

All continuous variables were changed to categorical variables. Pearson $\chi^{2}$ test was used to compute the $P$-value. 


\begin{tabular}{|c|c|c|c|}
\hline No & $21(7.0)$ & $13(7.1)$ & \\
\hline Yes & 170 (56.7) & $100(54.9)$ & \\
\hline Reoperation & 109 (36.3) & 69 (37.9) & \\
\hline Radiotherapy & & & $<0.001$ \\
\hline No & 179 (59.7) & $129(70.9)$ & \\
\hline $2 \mathrm{D}$ & $22(7.3)$ & $22(12.1)$ & \\
\hline IMRT & $99(33.0)$ & 31 (17.0) & \\
\hline Chemotherapy & & & 0.377 \\
\hline No & $131(43.7)$ & $87(47.8)$ & \\
\hline Yes & 169 (56.3) & $95(52.2)$ & \\
\hline Immunotherapy & & & 0.659 \\
\hline No & 217 (72.3) & 135 (74.2) & \\
\hline Yes & 83 (27.7) & $47(25.8)$ & \\
\hline Abbreviations: & & & \\
\hline
\end{tabular}

The median follow-up time was 32.0 months (range, 1-262 months). A total of 172 patients experienced disease failure; 75 (7.7\%), 52 (3.8\%), and 109 (14.6\%) patients developed local recurrence, regional recurrence, and distant metastasis, respectively; and 188 (14.4\%) patients died.

\section{Univariate and Multivariate Cox Regression Analyses}

The significant variables related to OS were primary tumor location, T stage, $\mathrm{N}$ stage, and immunotherapy in univariate analysis. The significant variables related to DFS, DMFS, and LRRFS were primary tumor location, $\mathrm{T}$ stage, and $\mathrm{N}$ stage in univariate analysis. We incorporated the above factors into multivariate Cox regression analysis. Eventually, $\mathrm{T}$ stage, $\mathrm{N}$ stage, and primary tumor site were independent prognostic factors. The results of univariate and multivariate Cox analyses are summarized in Table 2, 3 and Fig. 1. 
Table 2

Univariate Cox regression analysis of OS and DFS in the primary cohort.

\begin{tabular}{|c|c|c|c|c|}
\hline \multirow[t]{2}{*}{ Variable } & \multicolumn{2}{|l|}{ OS } & \multicolumn{2}{|l|}{ DFS } \\
\hline & $\mathrm{HR}(95 \% \mathrm{Cl})$ & $\mathbf{P}$ & $\mathrm{HR}(95 \% \mathrm{Cl})$ & $\mathbf{P}$ \\
\hline \multicolumn{5}{|l|}{ Gender } \\
\hline Male & Reference & & Reference & \\
\hline Female & 0.964 (0.717 to 1.297$)$ & 0.811 & $\begin{array}{l}1.051 \text { ( } 0.766 \text { to } \\
1.442)\end{array}$ & 0.759 \\
\hline \multicolumn{5}{|l|}{ Age (years old) } \\
\hline$<60$ & Reference & & Reference & \\
\hline$\geq 60$ & $1.246(0.934$ to 1.660$)$ & 0.134 & $\begin{array}{l}1.203(0.881 \text { to } \\
1.644)\end{array}$ & 0.245 \\
\hline \multicolumn{5}{|l|}{ Smoking } \\
\hline No & Reference & & Reference & \\
\hline Yes & $1.020(0.747$ to 1.395$)$ & 0.899 & $\begin{array}{l}0.787 \text { ( } 0.591 \text { to } \\
1.049)\end{array}$ & 0.102 \\
\hline \multicolumn{5}{|l|}{ Primary site } \\
\hline Others & Reference & & Reference & \\
\hline Nasal cavity & 2.127 (1.256 to 3.603$)$ & 0.005 & $\begin{array}{l}1.510(0.996 \text { to } \\
2.288)\end{array}$ & 0.052 \\
\hline Paranasal sinus & $\begin{array}{l}6.113(3.101 \text { to } \\
12.052)\end{array}$ & $<.001$ & $\begin{array}{l}3.066 \text { (1.733 to } \\
5.425)\end{array}$ & $\hat{L}_{0.001}$ \\
\hline Oral cavity & $2.586(1.465$ to 4.564$)$ & 0.001 & $\begin{array}{l}2.217 \text { ( } 1.413 \text { to } \\
3.479)\end{array}$ & 0.001 \\
\hline \multicolumn{5}{|l|}{ 8th T stage } \\
\hline Т3 & Reference & & Reference & \\
\hline T4a & 2.801 (1.968 to 3.989 ) & $\begin{array}{l}< \\
0.001\end{array}$ & $\begin{array}{l}2.150(1.596 \text { to } \\
2.896)\end{array}$ & ¿. 001 \\
\hline $\mathrm{T} 4 \mathrm{~b}$ & $\begin{array}{l}11.025(7.301 \text { to } \\
16.647)\end{array}$ & $\hat{0} .001$ & $\begin{array}{l}6.179 \text { ( } 4.227 \text { to } \\
9.034)\end{array}$ & 广. 001 \\
\hline \multicolumn{5}{|l|}{ 8th N stage } \\
\hline NO & Reference & & Reference & \\
\hline N1 & 1.542 (1.113 to 2.237$)$ & 0.009 & $\begin{array}{l}1.637 \text { (1.223 to } \\
2.191)\end{array}$ & 0.001 \\
\hline
\end{tabular}




\begin{tabular}{|c|c|c|c|c|}
\hline \multirow[t]{2}{*}{ Variable } & \multicolumn{2}{|l|}{ os } & \multicolumn{2}{|l|}{ DFS } \\
\hline & $\mathrm{HR}(95 \% \mathrm{Cl})$ & $\mathbf{P}$ & $\mathrm{HR}(95 \% \mathrm{Cl})$ & $\mathbf{P}$ \\
\hline \multicolumn{5}{|l|}{ Surgery } \\
\hline No & Reference & & Reference & \\
\hline Yes & 0.707 (0.409 to 1.220$)$ & 0.212 & $\begin{array}{l}0.612(0.386 \text { to } \\
0.969)\end{array}$ & 0.036 \\
\hline \multicolumn{5}{|c|}{ Radiotherapy } \\
\hline No & Reference & & Reference & \\
\hline Yes & 1.178 (0.879 to 1.580$)$ & 0.273 & $\begin{array}{l}1.070 \text { (0.828 to } \\
1.383)\end{array}$ & 0.603 \\
\hline \multicolumn{5}{|c|}{ Chemotherapy } \\
\hline No & Reference & & Reference & \\
\hline Yes & 0.863 (0.647 to 1.151$)$ & 0.317 & $\begin{array}{l}1.022(0.792 \text { to } \\
1.318)\end{array}$ & 0.869 \\
\hline \multicolumn{5}{|c|}{$\begin{array}{l}\text { Immunologic/targeted } \\
\text { therapy }\end{array}$} \\
\hline No & Reference & & Reference & \\
\hline Yes & 0.694 (0.495 to 0.974$)$ & 0.035 & $\begin{array}{l}0.873 \text { ( } 0.657 \text { to } \\
1.159)\end{array}$ & 0.348 \\
\hline
\end{tabular}


Table 3

Univariate Cox regression analysis of DMFS and LRRFS in the primary cohort.

\begin{tabular}{|c|c|c|c|c|}
\hline \multirow[t]{2}{*}{ Variable } & \multicolumn{2}{|l|}{ DMFS } & \multicolumn{2}{|l|}{ LRRFS } \\
\hline & $\mathrm{HR}(95 \% \mathrm{Cl})$ & $\mathbf{P}$ & $\mathrm{HR}(95 \% \mathrm{Cl})$ & $\mathbf{P}$ \\
\hline \multicolumn{5}{|l|}{ Gender } \\
\hline Male & Reference & & Reference & \\
\hline Female & 0.964 (0.717 to 1.297$)$ & 0.811 & $\begin{array}{l}1.036(0.793 \text { to } \\
1.355)\end{array}$ & 0.794 \\
\hline \multicolumn{5}{|l|}{ Age (years old) } \\
\hline$<60$ & Reference & & Reference & \\
\hline$\geq 60$ & $1.246(0.641$ to 1.164$)$ & 0.134 & $\begin{array}{l}1.070(0.822 \text { to } \\
1.391)\end{array}$ & 0.616 \\
\hline \multicolumn{5}{|l|}{ Smoking } \\
\hline No & Reference & & Reference & \\
\hline Yes & $0.864(0.625$ to 1.236$)$ & 0.336 & $\begin{array}{l}0.819(0.609 \text { to } \\
1.100)\end{array}$ & 0.185 \\
\hline \multicolumn{5}{|l|}{ Primary site } \\
\hline Others & Reference & & Reference & \\
\hline Nasal cavity & 1.695 (1.084 to 2.649$)$ & 0.021 & $\begin{array}{l}1.795 \text { (1.128 to } \\
2.858)\end{array}$ & 0.014 \\
\hline Paranasal sinus & 3.736 (2.032 to 6.867$)$ & $\begin{array}{l}<.001 \\
0.01\end{array}$ & $\begin{array}{l}3.710(1.995 \text { to } \\
6.897)\end{array}$ & <. 001 \\
\hline Oral cavity & $\begin{array}{l}1.9844 \text { (1.217 to } \\
3.235)\end{array}$ & 0.006 & $\begin{array}{l}2.631 \text { (1.601 to } \\
4.323)\end{array}$ & <. 001 \\
\hline \multicolumn{5}{|l|}{ 8th T stage } \\
\hline Т3 & Reference & & Reference & \\
\hline T4a & $2.544(1.838,3.520)$ & $\begin{array}{l}<.001 \\
0.01\end{array}$ & $\begin{array}{l}1.945(1.434 \text { to } \\
2.639)\end{array}$ & <. 001 \\
\hline T4b & $10.314(6.861,15.502)$ & <.001 & $\begin{array}{l}5.816 \text { (4.002 to } \\
8.452)\end{array}$ & $\langle .001$ \\
\hline \multicolumn{5}{|l|}{ 8th N stage } \\
\hline NO & Reference & & Reference & \\
\hline N1 & 1.597 (1.180 to 2.161$)$ & 0.002 & $\begin{array}{l}1.665 \text { (1.231 to } \\
2.253)\end{array}$ & 0.001 \\
\hline
\end{tabular}




\begin{tabular}{|c|c|c|c|c|}
\hline \multirow[t]{2}{*}{ Variable } & \multicolumn{2}{|l|}{ DMFS } & \multicolumn{2}{|l|}{ LRRFS } \\
\hline & HR (95\% Cl) & $P$ & HR (95\% Cl) & $P$ \\
\hline \multicolumn{5}{|l|}{ Surgery } \\
\hline No & Reference & & Reference & \\
\hline Yes & $0.758(0.455$ to 1.262$)$ & 0.286 & $\begin{array}{l}0.664 \text { ( } 0.409 \text { to } \\
1.078)\end{array}$ & 0.098 \\
\hline \multicolumn{5}{|c|}{ Radiotherapy } \\
\hline No & Reference & & Reference & \\
\hline Yes & $1.359(1.037$ to 1.224$)$ & 0.026 & $\begin{array}{l}1.007 \text { (0.771 to } \\
1.316)\end{array}$ & 0.957 \\
\hline \multicolumn{5}{|c|}{ Chemotherapy } \\
\hline No & Reference & & Reference & \\
\hline Yes & $1.022(0.792$ to 1.318$)$ & 0.869 & $\begin{array}{l}0.934 \text { (0.718 to } \\
1.215)\end{array}$ & 0.611 \\
\hline \multicolumn{5}{|c|}{$\begin{array}{l}\text { Immunologic/targeted } \\
\text { therapy }\end{array}$} \\
\hline No & Reference & & Reference & \\
\hline Yes & $0.858(0.634$ to 1.161$)$ & 0.320 & $\begin{array}{l}0.801 \text { ( } 0.594 \text { to } \\
1.079)\end{array}$ & 0.144 \\
\hline
\end{tabular}

\section{Establishing and Validating a Nomogram}

All factors, including primary tumor site, $\mathrm{T}$ stage, and $\mathrm{N}$ stage, were included in the nomogram. By summarizing the score of each variable and positioning the total scores on the score scale, a nomogram was established to predict the 3- and 5-year OS, DFS, DMFS, and LRRFS in the primary cohort (Figs. 2AD).

The c-indexes of the nomogram for predicting OS, DFS, DMFS, and LRRFS were almost over 0.7 in all cohorts, which indicated that the performance of the model was satisfactory.

In the calibration plot, the X-axis is the nomogram prediction of OS, DFS, DMFS, and LRRFS, and the Yaxis is the observed values of OS, DFS, DMFS, and LRRFS calculated by the Kaplan-Meier method. The solid line is the ideal reference line, which represents the consistency between the predicted survival rate and the observed survival rate. The calibration plots for the OS, DFS, DMFS, and LRRFS probabilities at 3 and 5 years showed significant correspondence between the predictions and observations in all cohorts (Figs. 3 and 4). Moreover, ROC curve analysis was also used to assess the predictive capacity of the nomogram, and the AUC for all cohorts indicated excellent validity (AUC >0.70) (Fig. 5). 


\section{Nomogram for Risk Stratification}

Consequently, stratification was established according to the nomogram for OS, DFS, DMFS, and LRRFS. Based on the total scores calculated by the nomogram, we set a threshold for the total score $(33 \%$ and $66 \%$ ). The primary and validation cohorts were divided into low-risk [total score: $<50$ (OS); $<42$ (DFS); $<40$ (DMFS); <45 (LRRFS)], intermediate-risk [total score: 50-85 (OS); 42-64 (DFS); 40-60 (DMFS); 45-69 (LRRFS)] and high-risk [total score: > 85 (OS); >64 (DFS); >60 (DMFS); >69 (LRRFS)] groups. According to the Kaplan-Meier survival curves, there were obvious differences among the different risk groups (all Pvalues< 0.001; Fig. 6).

\section{Establishment of a web server for Easy Application of the nomogram}

We have created a web server of our nomogram (Fig. 7), which can be easily accessed at https://liling.shinyapps.io/MMHN_OS/, https://liling.shinyapps.io/MMHN_DFS/, https://liling.shinyapps.io/MMHN_DMFS/, and https://liling.shinyapps.io/MMHN_LRRFS/. OS, DFS, DMFS, and LRRFS in NPC patients can be predicted conveniently by selecting corresponding clinical features and reading generated figures and tables.

\section{Discussion}

MMHN is an aggressive malignancy whose comprehensive treatment modalities are complex and clinical outcomes are still unsatisfactory ${ }^{12}$. The AJCC 8 th edition staging system lacks the ability to predict prognosis ${ }^{13}$. MMHN patients in the same stage can have obviously different survival rates ${ }^{14}$. Integrating other predictive factors into the TNM staging system will add prognostic value to this system and guide more promising treatment decisions.

Our results revealed that the primary tumor site was an independent prognostic factor. Consistent with the findings of several previous studies, our results showed that worse outcomes existed when the primary tumor site was in the paranasal cavity than in the oral or nasal cavities ${ }^{15-17}$. There may be several plausible reasons for the poorer survival of patients with a primary tumor site in the paranasal sinuses. Due to the complex anatomical location of the paranasal sinus, paranasal sinus melanoma is found at later stages and has a wide range of invasion, so the prognosis of these patients may be poor. $T$ stage and $\mathrm{N}$ stage were also independent prognostic factors, similar to the findings of other articles ${ }^{18-20}$.

The study has the following advantages. First, the survival rate of a patient can be calculated simply and visually. For example, suppose a patient with T3 (0 points) N0 (0 points) paranasal sinus MM (50 points) comes to the clinic; he/she would have a total of 50 points, yielding an estimated 3-year OS rate of $28.57 \%$. Second, the nomogram can further divide patients into high-, intermediate- and low-risk groups, and the survival rate of each group can effectively distinguish the prognosis. Doctors can give individualized treatment to patients according to their corresponding groups. The intensity of treatment 
can be appropriately strengthened for patients in the high-risk group. For example, immunologic/targeted therapy can improve the survival rate of $\mathrm{MMHN}^{21}$. Finally, in addition to the traditional nomogram, we also created a dynamic nomogram that can predict the prognosis of patients through simple operation on a web page (Fig. 7).

Our study has several limitations. First, this study was a retrospective study with inevitable selection bias. However, this retrospective study was worth carrying out because it is of great significance to lay the foundation for further prospective studies.

Second, this study involved patients from the same hospital; thus, the findings may lack applicability for patients from other regions and institutions. Finally, the sample size was not large enough, so there were no significant differences in some prognostic factors, especially the treatment modalities. Nonetheless, our study included 300 Chinese MMHN patients, which currently constitutes the largest sample from a single center to our knowledge.

In conclusion, our nomogram combines primary site with $\mathrm{T}$ stage and $\mathrm{N}$ stage to predict the survival outcomes of MMHN patients, which can help doctors easily distinguish high- and low-risk patients clinically for individualized treatment.

\section{Abbreviations}

AJCC/UICC: American Joint Committee on Cancer/Union for International Cancer Control; Cl: confidence interval; OS: overall survival; DMFS: distant metastasis-free survival; HR: hazard ratio; IMRT: intensitymodulated radiotherapy; LRRFS: locoregional relapse-free survival.

\section{Declarations}

\section{Acknowledgements}

Not applicable

\section{Authors' contributions}

Conceptualization: LXL, LC, QQX. Data analysis: QQX, QJL, LC, XYS. Original draft writing: QQX, QJL, LC, JXS. Review and editing: LXL, LC, QQX, QJL, XYS, JXS. All authors read and approved the final manuscript.

\section{Funding}

This study was funded by the Planned Science and Technology Project of Guangdong Province (No 2016A020215085, 201707010087) and the 308 Clinical Research Funding of Sun Yat-Sen University Cancer Center (No 308-2015-011). 
Availability of data and materials

The databases analyzed during the current study are available.

\section{Ethics approval and consent to participate}

The Hospital Ethics Committee at Sun Yat-sen University Cancer Center in China

approved the study, which analyzed anonymous information as well as waived the demand for informed consent.

\section{Consent for publication}

Not applicable.

\section{Competing interests}

The authors declare that they have no competing interests.

\section{Author details}

${ }^{1}$ Department of Radiation Oncology, Sun Yat-sen University Cancer Center; State Key Laboratory of Oncology in South China; Collaborative Innovation Center for Cancer Medicine; Guangdong Key Laboratory of Nasopharyngeal Carcinoma Diagnosis and Therapy, Guangzhou 510060, China

${ }^{2}$ Department of Radiation Oncology, Hubei Cancer Hospital, Tongji Medical College, Huazhong University of Science and Technology, Wuhan 430079, Hubei, P.R. China

\section{References}

1. Chang AE, Karnell LH, Menck HR. The National Cancer Data Base report on cutaneous and noncutaneous melanoma: a summary of 84,836 cases from the past decade. The American College of Surgeons Commission on Cancer and the American Cancer Society. Cancer. 1998;83:1664-78.

2. Hicks MJ, Flaitz CM. Oral mucosal melanoma: epidemiology and pathobiology. Oral Oncol. 2000;36:152-69.

3. Furney SJ, Turajlic S, Stamp G, et al. Genome sequencing of mucosal melanomas reveals that they are driven by distinct mechanisms from cutaneous melanoma. J Pathol. 2013;230:261-9.

4. Mendenhall WM, Amdur RJ, Hinerman RW, Werning JW, Villaret DB, Mendenhall NP. Head and neck mucosal melanoma. Am J Clin Oncol. 2005;28:626-30.

5. Lazarev S, Gupta V, Hu K, Harrison LB, Bakst R. Mucosal melanoma of the head and neck: a systematic review of the literature. Int J Radiat Oncol Biol Phys. 2014;90:1108-18.

6. Ascierto PA, Accorona R, Botti G, et al. Mucosal melanoma of the head and neck. Crit Rev Oncol Hematol. 2017;112:136-52. 
7. McLean N, Tighiouart M, Muller S. Primary mucosal melanoma of the head and neck. Comparison of clinical presentation and histopathologic features of oral and sinonasal melanoma. Oral Oncol. 2008;44:1039-46.

8. Wang Y, Li J, Xia Y, et al. Prognostic nomogram for intrahepatic cholangiocarcinoma after partial hepatectomy. J Clin Oncol. 2013;31:1188-95.

9. Kim Y, Margonis GA, Prescott JD, et al. Nomograms to Predict Recurrence-Free and Overall Survival After Curative Resection of Adrenocortical Carcinoma. JAMA Surg. 2016;151:365-73.

10. Sun X-S, Liang Y-J, Liu S-L, et al. Establishment and validation of a nomogram for predicting survival in patients with de novo metastatic nasopharyngeal carcinoma. Oral Oncol. 2019;94:73-9.

11. Dess RT, Suresh K, Zelefsky MJ, et al. Development and Validation of a Clinical Prognostic Stage Group System for Nonmetastatic Prostate Cancer Using Disease-Specific Mortality Results From the International Staging Collaboration for Cancer of the Prostate. JAMA Oncol. 2020;6:1912-20.

12. Chan RC-L, Chan JYW, Wei WI. Mucosal melanoma of the head and neck: 32-year experience in a tertiary referral hospital. Laryngoscope. 2012;122:2749-53.

13. Flukes S, Lohia S, Barker CA, et al. Primary tumor volume as a predictor of distant metastases and survival in patients with sinonasal mucosal melanoma. Head Neck. 2020;42:3316-25.

14. Dejaco D, Url C, Schartinger VH, et al. Approximation of head and neck cancer volumes in contrast enhanced CT. Cancer Imaging. 2015;15:16.

15. Samstein RM, Carvajal RD, Postow MA, et al. Localized sinonasal mucosal melanoma: Outcomes and associations with stage, radiotherapy, and positron emission tomography response. Head Neck. 2016;38:1310-7.

16. Khan MN, Kanumuri VV, Raikundalia MD, et al. Sinonasal melanoma: survival and prognostic implications based on site of involvement. Int Forum Allergy Rhinol. 2014;4:151-5.

17. Roth TN, Gengler C, Huber GF, Holzmann D. Outcome of sinonasal melanoma: clinical experience and review of the literature. Head Neck. 2010;32:1385-92.

18. Sun S, Huang X, Gao L, et al. Long-term treatment outcomes and prognosis of mucosal melanoma of the head and neck: 161 cases from a single institution. Oral Oncol. 2017;74:115-22.

19. Schmidt MQ, David J, Yoshida EJ, et al. Predictors of survival in head and neck mucosal melanoma. Oral Oncol. 2017;73:36-42.

20. Moya-Plana A, Aupérin A, Obongo R, et al. Oncologic outcomes, prognostic factor analysis and therapeutic algorithm evaluation of head and neck mucosal melanomas in France. Eur $\mathrm{J}$ Cancer $2019 ; 123$.

21. van Zeijl MCT, Boer FL, van Poelgeest MIE, et al. Survival outcomes of patients with advanced mucosal melanoma diagnosed from 2013 to 2017 in the Netherlands - A nationwide populationbased study. Eur J Cancer. 2020;137:127-35.

\section{Figures}


A

Variable

Primary site (vs Others)

Nasal cavity

Paranasal sinus

Oral cavity

8th T stage (vs T3)

$\mathrm{T} 4 \mathrm{a}$

$\mathrm{T} 4 \mathrm{~b}$

8th $\mathrm{N}$ stage (N1 vs NO)

Immunotherapy (Yes vs No)

B

Variable

Primary site (vs Others)

Nasal cavity

Paranasal sinus

Oral cavity

8th T stage (vs T3)

$\mathrm{T} 4 \mathrm{a}$

$\mathrm{T} 4 \mathrm{~b}$

8th N stage (N1 vs N0)

C

Variable

Primary site (vs Others)

Nasal cavity

Paranasal sinus

Oral cavity

8th T stage (vs T3)

$\mathrm{T} 4 \mathrm{a}$

T4b

8th N stage (N1 vs N0)

D

Variable

Primary site (vs Others)

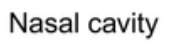

Paranasal sinus

Oral cavity

8th T stage (vs T3)

T4a

T4b

8th N stage (N1 vs N0)
HR $(95 \% \mathrm{Cl})$

$p$ value

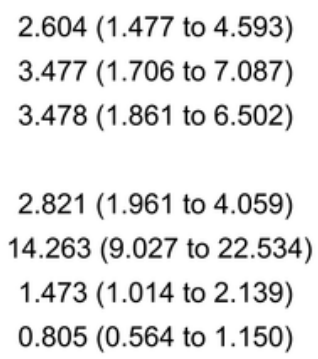

HR $(95 \% \mathrm{Cl})$

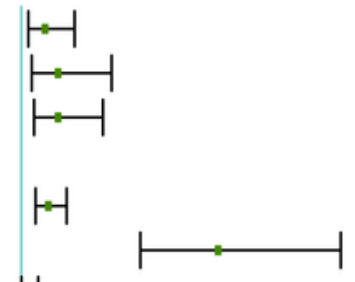

바

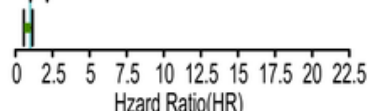
Hzard Ratio(HR)

$p$ value

1.480 (0.975 to 2.247$)$

1.526 (0.845 to 2.755$)$

2.099 (1.313 to 3.358 )

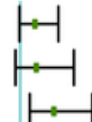

0.066

0.161

0.002

2.224 (1.641 to 3.015 )

6.695 (4.474 to 10.019 )

1.493 (1.081 to 2.061 )

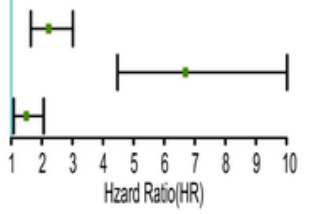

$<0.001$

$<0.001$

0.015

HR (95\% Cl)

1.647 (1.05 to 2.584$)$

1.645 (0.876 to 3.090$)$

1.849 (1.106 to 3.091 )

2.555 (1.831 to 3.565$)$

11.179 (7.252 to 17.232$)$

1.646 (1.168 to 2.319 )
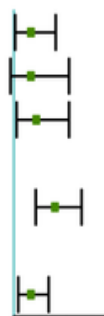

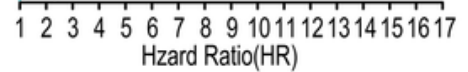

$\mathrm{HR}(95 \% \mathrm{Cl})$

p value

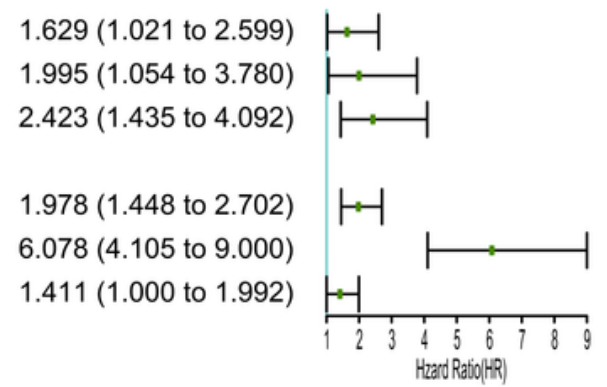

0.040

0.034

0.001

$<0.001$

$<0.001$

0.050
0.001

0.001

$<0.001$

$<0.001$

$<0.001$

0.042

0.233

\section{e}


A

Points

Primary site

8th T stage

8th $\mathrm{N}$ stage

Total points

3-year OS

5-year OS

Points

Primary site

8th T stage

8th $\mathrm{N}$ stage

Total points

3-year DFS

5-year DFS

C

Points

Primary site

8th T stage

8th $\mathrm{N}$ stage

Total points

3-year DMFS

5-year DMFS

Points

Primary site

8th T stage

8th $\mathrm{N}$ stage

Total Points

3-year LRRF

5-year LRRFS

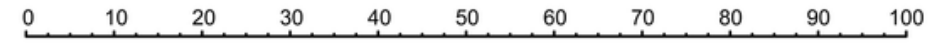

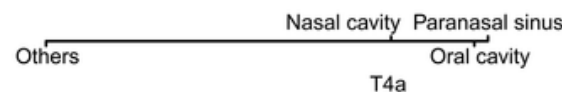

$T 4 a$

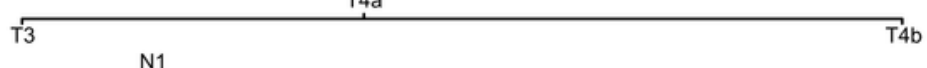

N1

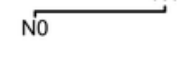

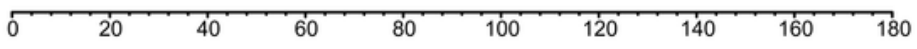$$
\begin{array}{lllllllll}
0.9 & 0.8 & 0.7 & 0.6 & 0.5 & 0.4 & 0.3 & 0.2 & 0.1
\end{array}
$$$$
\begin{array}{llllllll}
0.8 & 0.7 & 0.6 & 0.5 & 0.4 & 0.3 & 0.2 & 0.1
\end{array}
$$

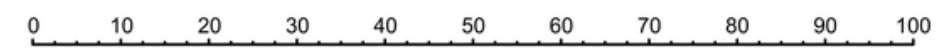
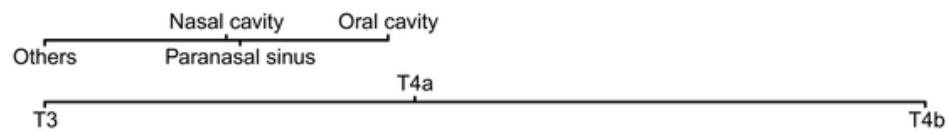

$$
\text { No }
$$

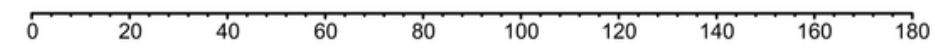

\begin{tabular}{lllllll}
\hline 0.7 & 0.6 & 0.5 & 0.4 & 0.3 & 0.2 & 0.1
\end{tabular}

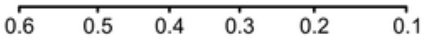

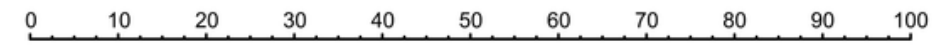
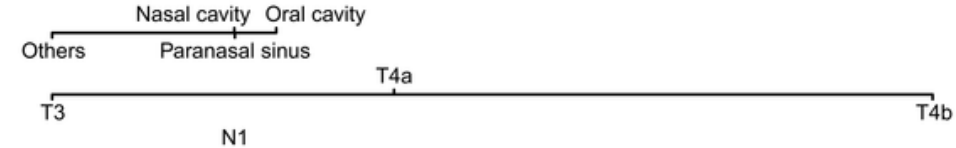

$$
\text { No }
$$

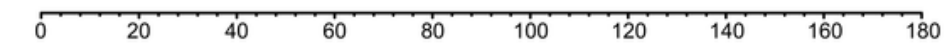

$\begin{array}{llllllll}0.8 & 0.7 & 0.6 & 0.5 & 0.4 & 0.3 & 0.2 & 0.1\end{array}$

$\begin{array}{lllllll}0.7 & 0.6 & 0.5 & 0.4 & 0.3 & 0.2 & 0.1\end{array}$
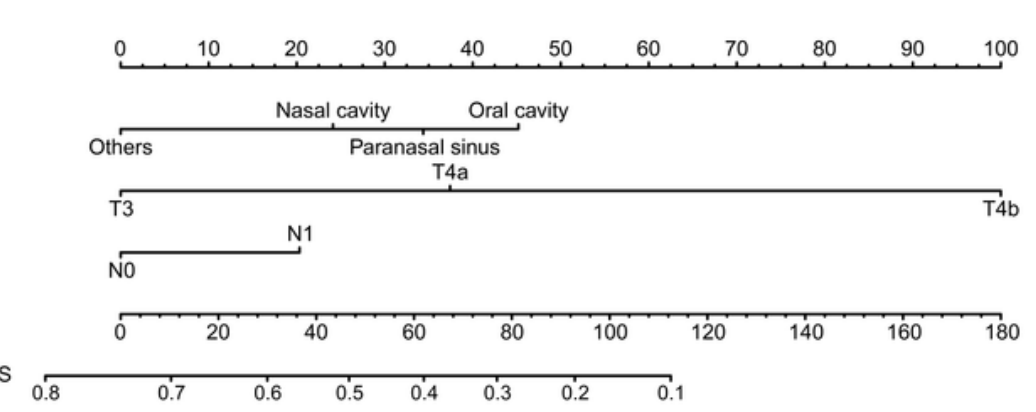

$\begin{array}{llllll}0.6 & 0.5 & 0.4 & 0.3 & 0.2 & 0.1\end{array}$

Figure 2

Developing a nomogram. Nomogram was based on the NPC patients' data of primary site, 8th T stage, and 8th N stage for 3-, 5-year OS (A), DFS (B), DMFS (C), and LRRFS (D) in the primary cohort. 

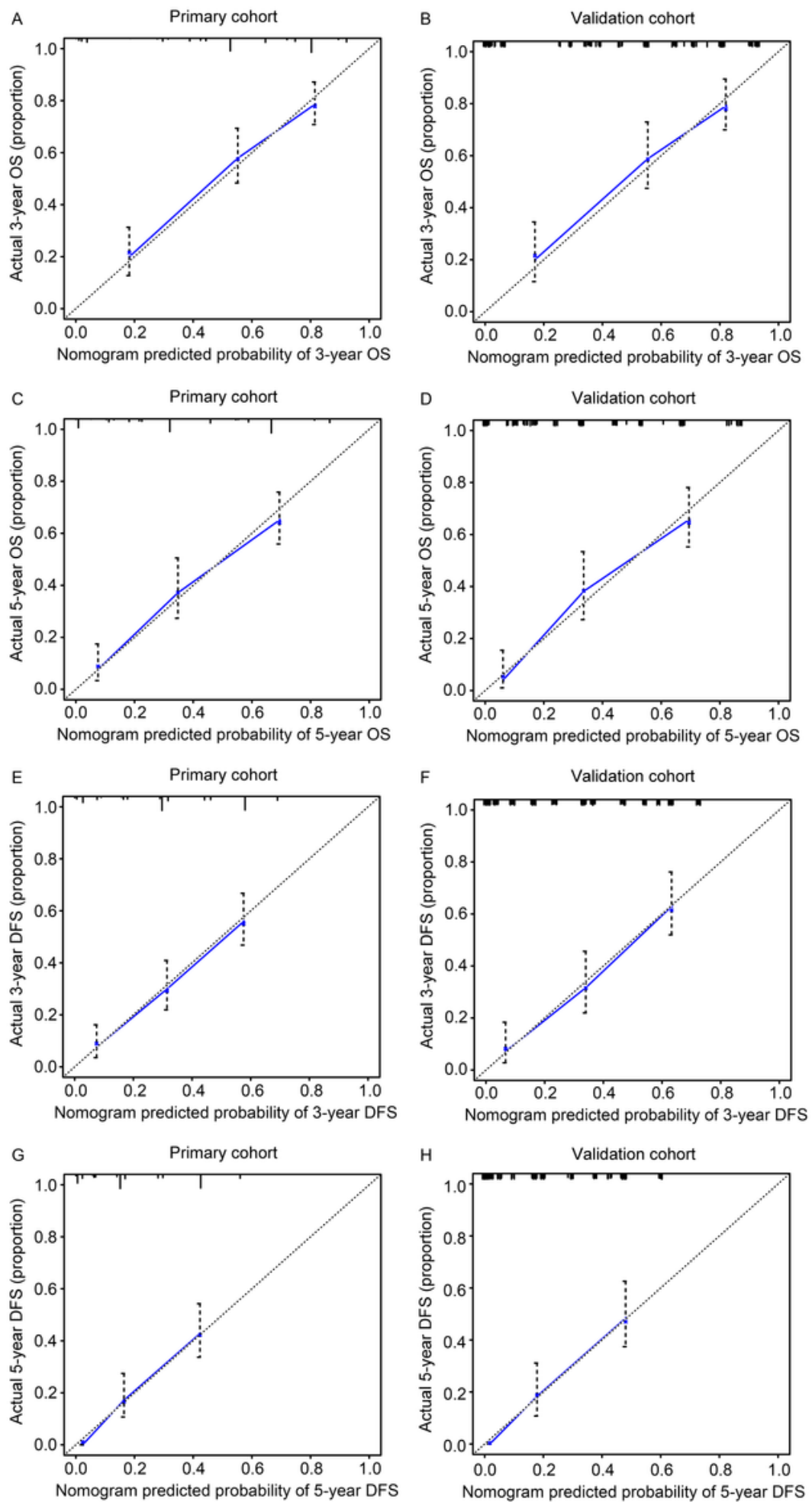

Figure 3

Calibration plots of the nomogram to predict 3- and 5-year OS and DFS in the primary $(A, C, E, G)$ and validation cohorts $(B, D, F, H)$. Nomogram-estimated 3- or 5-year OS (A-D) and DFS $(E-H)$ were plotted on the $x$-axis; the observed OS and DFS were plotted on the y-axis. Dashed lines along the 45-degree line represented that the predicted probabilities are equal to the actual probabilities. 

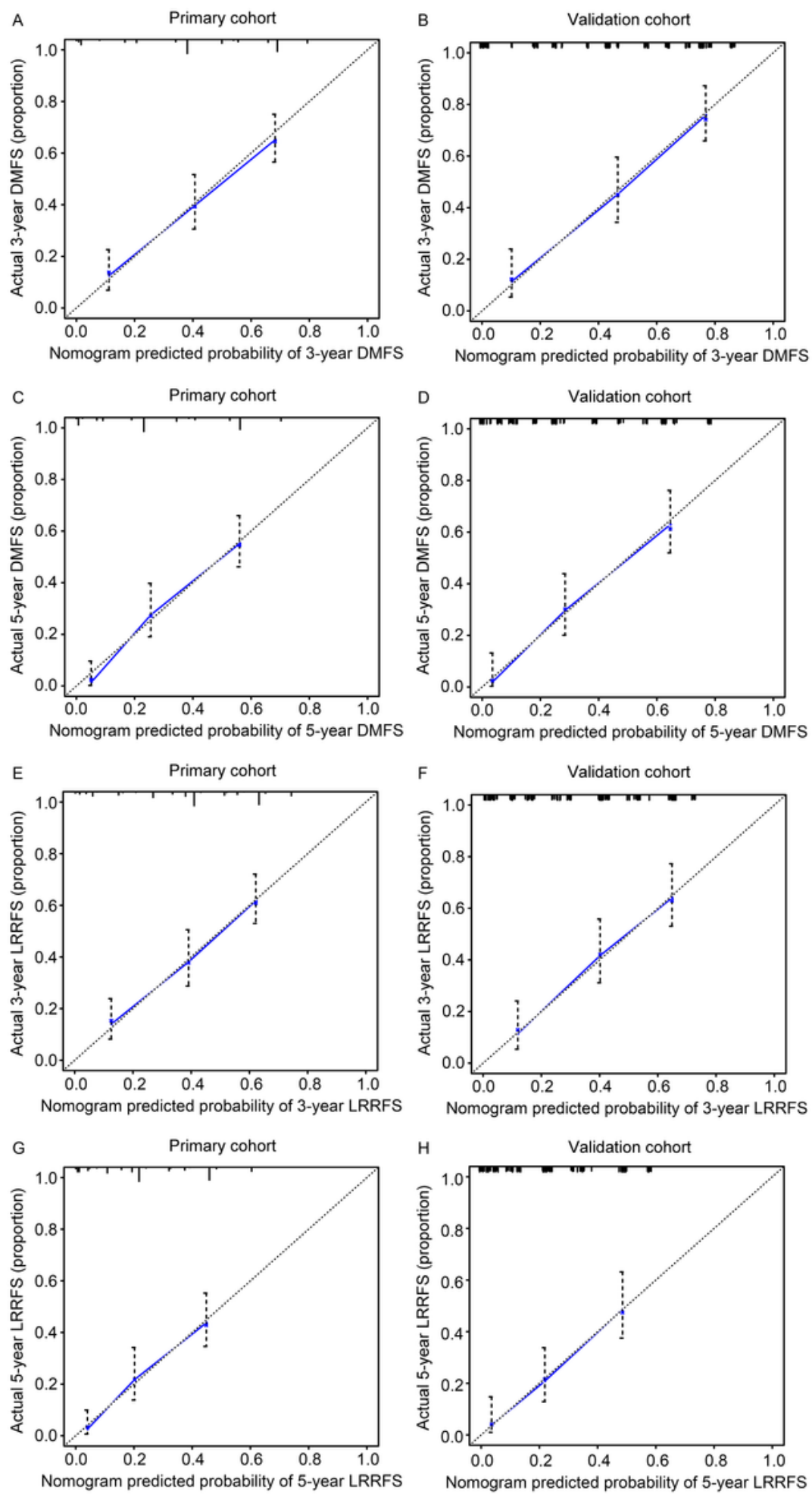

\section{Figure 4}

Calibration plots for 3- and 5-year DMFS and LRRFS in the primary $(A, C, E, G)$ and validation cohorts (B, $D, F, H)$. Nomogram-estimated 3- or 5-year DMFS (A-D) and LRRFS (E-H) were plotted on the x-axis; the observed DMFS and LRRFS were plotted on the $y$-axis. Dashed lines along the 45-degree line represented that the predicted probabilities are equal to the actual probabilities. 
A
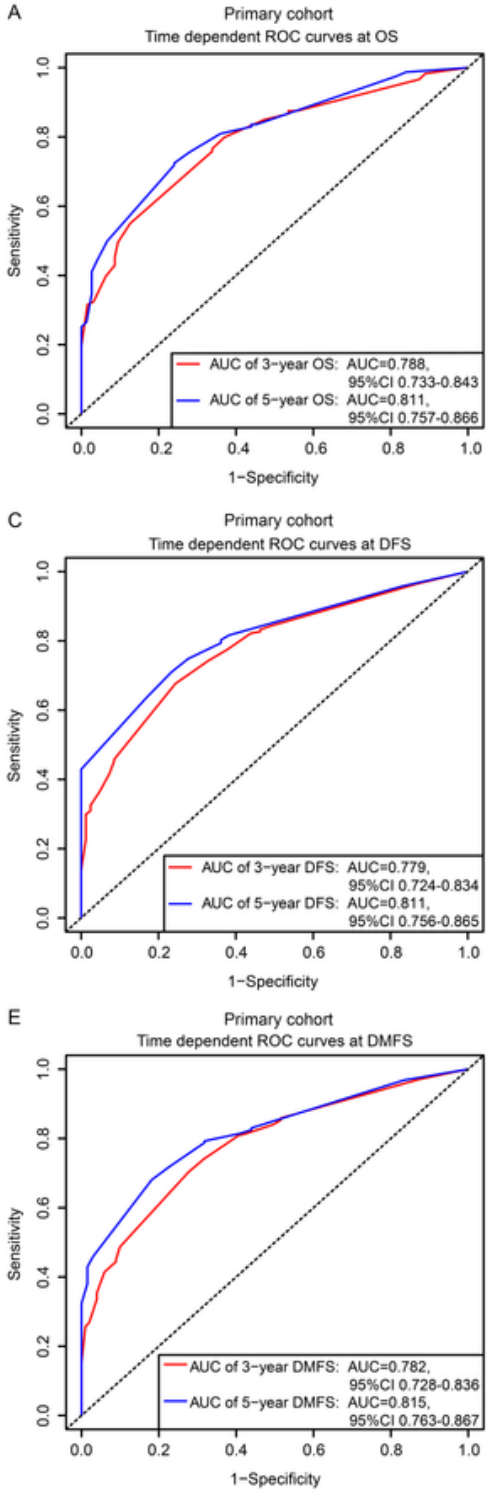

F

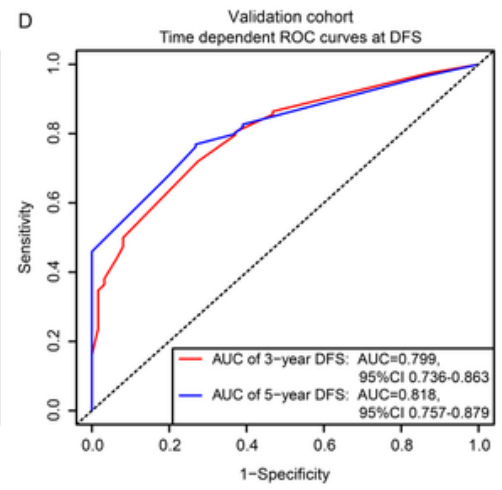

Validation cohort

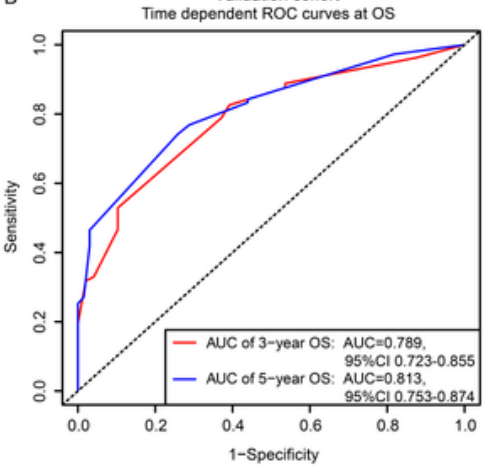

D

Validation cohort

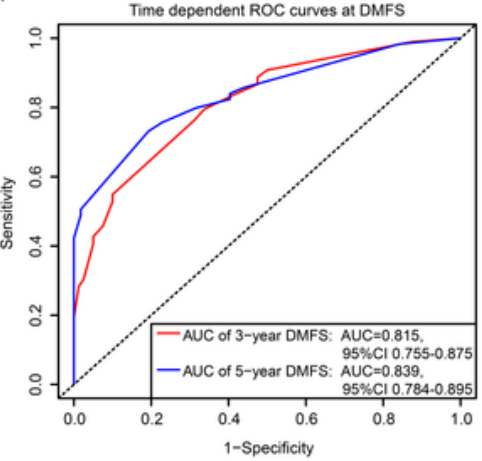

$\mathrm{H}$
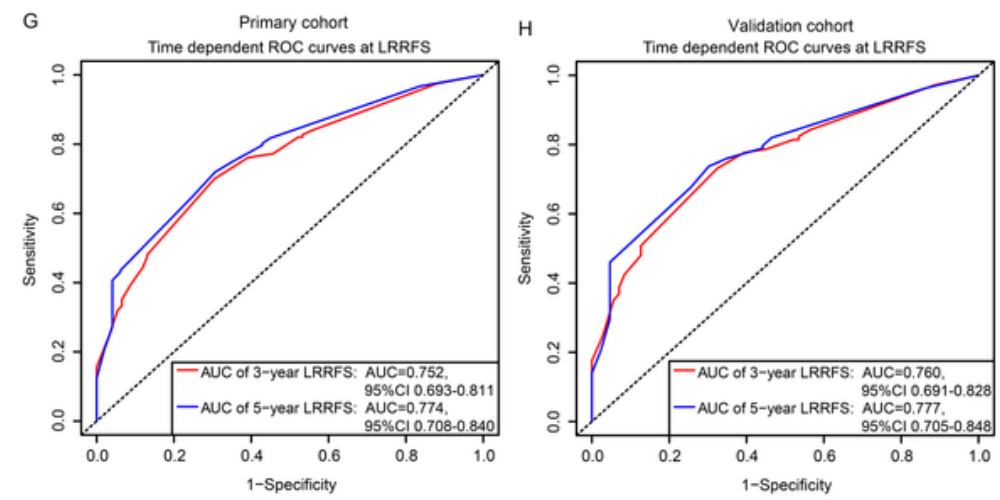

\section{Figure 5}

ROC curves by the nomogram to predict 3-, 5-year OS (A, B), DFS (C, D), DMFS $(E, F)$, and LRRFS $(G, H)$. 
A

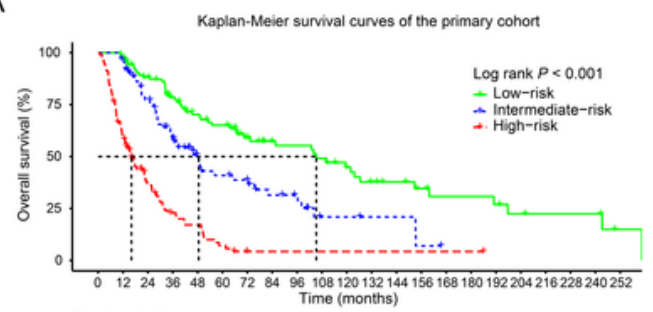

Number at risk

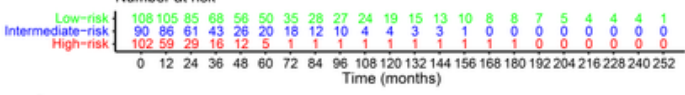

C

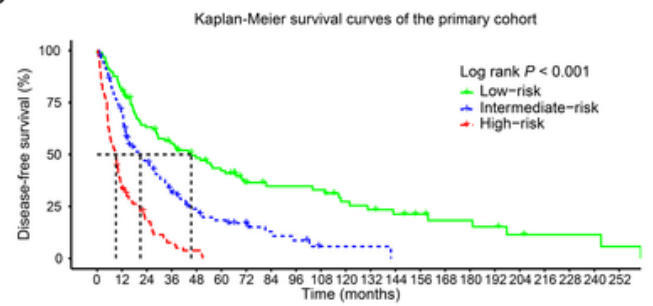

Number at risk

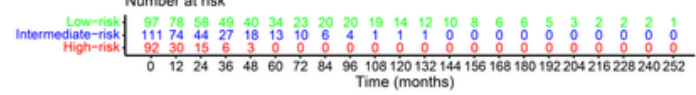

E

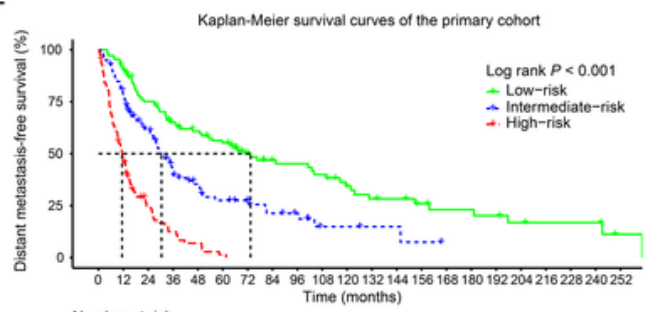

Number at risk

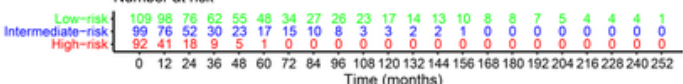

G

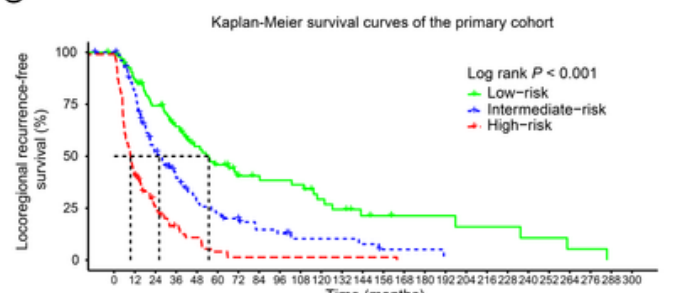

Number at risk

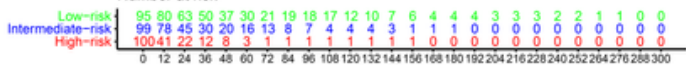

B

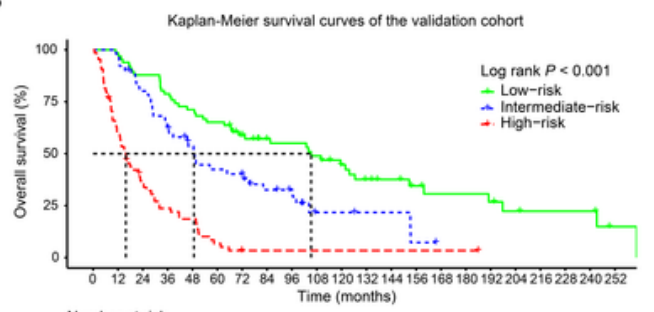

Number at risk

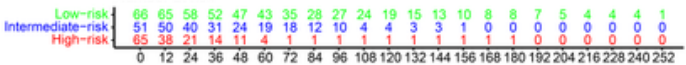

D

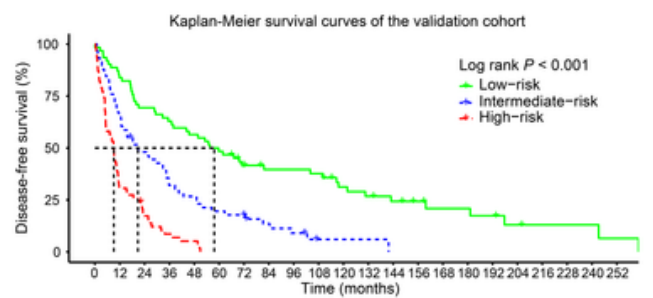

Number at risk

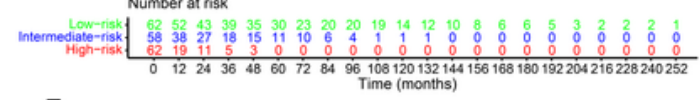

F

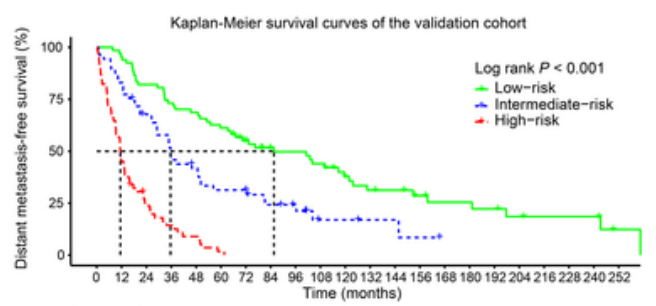

Number at risk

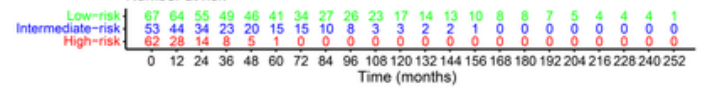

म

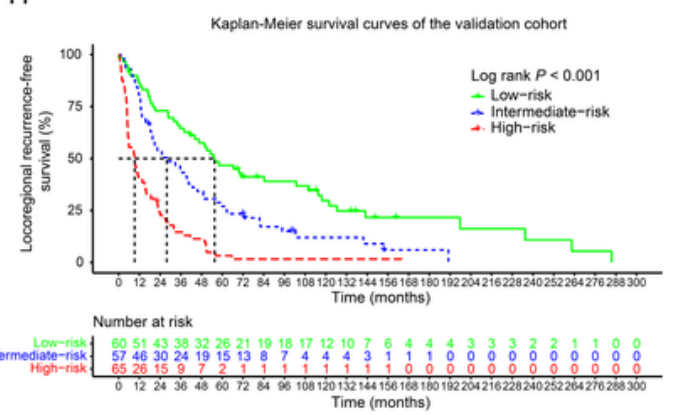

\section{Figure 6}

Kaplan-Meier curves for OS (A, B), DFS (C, D), DMFS (E, F), and LRRFS $(G, H)$ of patients in low-, intermediate- and high-risk groups. The risk groups were stratified according to the $33 \%$ and $66 \%$ of total risk scores in the primary $(A, C, E, G)$ and validation cohorts $(B, D, F, H)$. 
Dynamic Nomogram

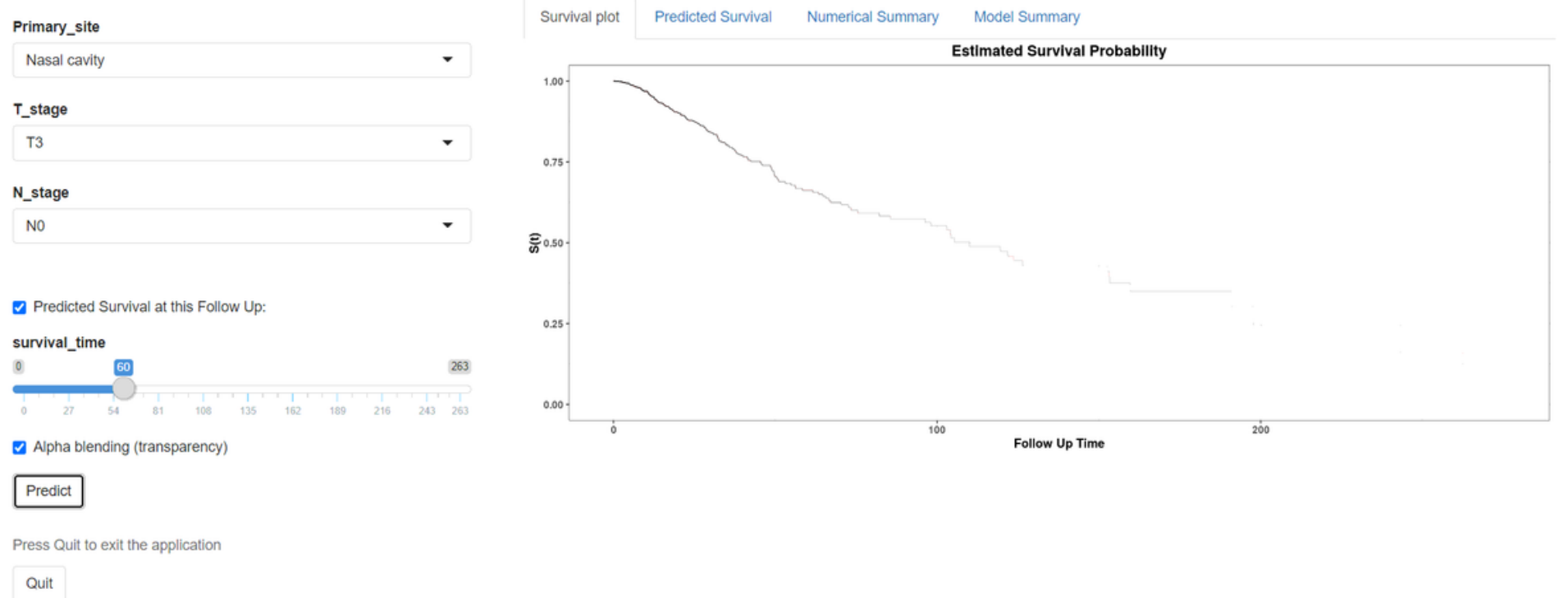

\section{Figure 7}

A web server for easy application of the nomogram 